\title{
Support Vector Machine Based Classification of Current Transformer Saturation Phenomenon
}

\author{
N. G. Chothani ${ }^{1}$, D. D. Patel $^{2}$ and K. D. Mistry ${ }^{2}$ \\ ${ }^{1}$ Electrical Department, A. D. Patel Institute of Technology, New Vallabh \\ Vidhyanagar, Gujarat, India \\ ${ }^{2}$ Electrical Department, Sardar Vallabhbhai National Institute of Technology, \\ Surat, Gujarat, India \\ E-mail: chothani_nilesh@rediffmail.com; ddeps2005@gmail.com; \\ kkp@eed.svnit.ac.in
}

Received 15 April 2017; Accepted 30 June 2017;

Publication 18 August 2017

\begin{abstract}
During out of zone fault, Current Transformer (CT) saturation leads maloperation in unit type protective schemes. Detection and classification of saturation condition of CT is still a challenging issue. Thus, it is most important to correctly categorize CT saturation condition to increase reliability and stability of protective schemes. The proposed scheme utilizes transmission line CT secondary post fault current signals (sliding window) as an input to SVM. In order to achieve the most optimized classifier, Gaussian Radial Basis Function (RBF) has been used for training of SVM. Feasibility of the proposed scheme has been tested by modelling a part of $220 \mathrm{kV}$ power systems in PSCAD/EMTDC software package. The algorithm is executed in MATLAB software. More than 720 unsaturated and 3600 saturated cases with varying burden resistance, remnant flux, DC component of current, noise penetration to current signal and fault inception angle have been generated and used for validation of the proposed scheme. The proposed scheme effectively discriminates between CT saturated and unsaturated conditions with very high classification accuracy more than $99 \%$ for different parameter variations.
\end{abstract}

Journal of Green Engineering, Vol. 7, 25-42.

doi: 10.13052/jge1904-4720.7122

(C) 2017 River Publishers. All rights reserved. 
Keywords: CT Saturation, Burden Resistance, Remnant Flux, Support Vector Machine (SVM), Power System, Fault Condition.

\section{Introduction}

Day by day power systems become more and more complicated, performance of protective relays encounters problems such as CT saturation and distorted current signal. Moreover, the quick improvement of protective technology has covered the way for the application of accurate and reliable methods in protective schemes. Presently, current differential relaying scheme is a proper protection for the single and multi-circuit protective means. However, this protection scheme faces difficulty in several situations. CT saturation attributable to an external fault may cause undesired trip of differential protection. In fact, this happen due to inequality in the burden of CTs or non-matching magnetizing features or remnant flux in one of the CTs at both ends.

In recent years, many researcher works have been dedicated to the problem of CT saturation detection and compensation but somehow all methods have its own limitations and until today proper methodology are not accepted in real field. Some papers have presented methods based on derivation of CT secondary current [1-6]. Ali Hooshyar et al. [1] presented nicely CT saturation detection based on wave shape properties of current difference functions, even they uses wave shape properties of current derivatives methodology on mathematical solution with all kind of possible situation like power factor, burden, fault inception angle etc. The accurate operation of this method is unaffected by the saturation severity level, noise, harmonics, and other transients even but practical implementation is remain left. Again Hooshyar et al. [2] developed a new derivative-based algorithm to detect current transformer saturation although detection time is large up to $200 \mathrm{~ms}$, it's so high. Chothani and Bhalja [3] suggested algorithm for current transformer saturation detection and compensation based on derivatives of secondary currents and Newton's backward difference formulae. This method is presented on mathematical base and validated but comparison with different method is remain left. Y. C. Kang et al. [4] explained design and evaluation of an algorithm for detecting current transformer saturation with a first order low-pass RC anti-aliasing filter. SangHee Kang et al. [5] presented a CT saturation detection algorithm based on wavelet transformation and a second order Butterworth high pass filter is used to extract the fine peak feature from the output signal of the filter banks. Filtering process of the DWT is just same methodology. D. Bak et al. [6] 
explained new method of detection of current transformer saturation using EMTP software analysis using first order derivatives of signals only. Moreover, there are papers which have proposed techniques based on decaying DC offset [7-9]. Ali Hooshyar and Majid Sanaye [7] elaborate CT saturation detection based on decaying DC offset in fault currents. Ehsan Hajipour et al. [8] explained CT saturation compensation for transformer differential relays based on Deformed Signal Compensation (DSC) and DC Offset Current Compensation (DOCC). T. Y. Ji et al. [9] proposed a morphological scheme for the correction of CT saturation waveforms using the estimation of the parameters of the DC offset with Genetic Algorithm (GA). Furthermore, Least Estimation Square (LES) based methods [10,11] are elaborated for CT saturation detection. F. B. Ajaei et al. [10] explained compensation of the current transformer saturation based on LES filtering technology for the current with Minimum Estimation Error Tracking (MEET) approach of the phasor estimation. D. Shi et al. [11] suggested fast compensation of current transformer saturation with computational of the estimation based on Separable Non Linear Least Squares (SNLLS) method. Meanwhile, phasor quantity based analysis are also carried out $[12,13]$ for CT saturation analysis. Ehab M. Esmail et al. [12] presented detection and compensation of current transformers by using Kalman filtering phasor quantities of the unsaturated current portion is extracted and basis of these phasor quantities, CT saturation is detected. N. Villamagna and P. Crossley [13] explained CT saturation detection using symmetrical components of current, phasor value of sequence component is estimated to detect the CT saturation. One another method for detection of CT saturation based on distance between two consecutive points [14, 15]. E. M. dos Santos et al. [14] proposed current transformer saturation detection based on the distance between consecutive points in the plans formed by the secondary current samples and their difference-functions. Chothani and Sharma [15] explained CT saturation detection using secondary current envelope detector using first order low pass filter.

Krzysztof Solak [16] et al. suggested fuzzy adaptive relaying scheme immune to CT saturation for avoiding maloperation of relaying scheme. However real hardware based implementation is complicated issue. Ali Hooshyar and M. Sanaye-Pasand $[17,18]$ elaborated wave shape recognition technique and variable-length window to detect current transformer saturation which is just same as [1] to analyze the current waveform, a new window designated as the Symmetry Assessment Window (SAW) is defined through hardware analysis but still improvement is required. J. G. Kappenman et al. [19] elaborated CT saturation based on Geomagnetically-Induced Currents (GIC) 
which is also utilizing as an effective scheme. On the other hand, effectiveness is a main constrain in various types of saturation effect. Bruno M. Schettino et al. [20] presented current-transformer saturation detection based on level of the Signal to Noise Ratio (SNR) and compared with the 3rd difference function method. However, the feasibility of the entire scheme for CT saturation mentioned above is not tested by considering variation in system and fault parameters.

In this paper, a new method for identification of saturated condition of current transformer using Support Vector Machine (SVM) is proposed by the authors. The proposed scheme provides effective discrimination between unsaturated and saturated condition. Feasibility of the proposed algorithm has been experienced by simulating various test cases using PSCAD/EMTDC software package [21]. The performance of the developed algorithm has been tested over a large data set (4320 simulation cases), generated using an automatic data generation model developed by the authors, considering wide variation in different system and fault conditions. The results show that the proposed algorithm provides well-organized classification for saturation of CT under various conditions.

\section{Problem Description}

Current transformer is the most important equipment in the power system protection. Saturation of a current transformer is one of the most burning issues in all protective schemes. Different conventional and intelligent methods are available to solve the problems of CT saturation in current transformer. Following issues detailed in Section 2.1 to 2.4 are accountable for saturation of CT in power system.

\subsection{Core over Sizing Factor}

For, well-organized design of core cross section of CT corresponds to $\phi_{a c}^{\max }$ should be near the knee point of B-H curve. To corresponding flux density (B) below the knee-point, one logical way to avoid CT saturation on DC flux is to oversize the core so, that flux $\left(\phi_{a c}^{\max }+\phi_{d c}^{\max }\right)$ passed through minimum remanence. Therefore, core over sizing factor is given by:

$$
\text { Core over sizing factor }=1+\frac{\phi_{d c}^{\max }}{\phi_{a c}^{\max }}=1+\frac{X}{R}
$$

As per Equation (1), the transmission line $\mathrm{X} / \mathrm{R}$ ratio for a $220 \mathrm{KV}$ line will be in the region of 7 to 10 and for $400 \mathrm{KV}$ line value of $\mathrm{X} / \mathrm{R}$ is approximately 20 . 
This would imply an over sizing required of about 21 times the usual design. Clearly, this high quantity of over sizing is not practical. Thus, power engineers have to overcome the saturation difficulty with constrain of CT size.

\subsection{Remnant Flux}

Secondly, the core saturation is a physical phenomenon. It happens when the coupled magnetic flux is so intense. That all magnetic domains on a ferromagnetic material are already aligned and thus does not respond to any further increase in the flux. The implications on the current transformer secondary current may be diverse. Having a saturated core does not mean the current on the CT secondary will be high or even constant as the flux increases. Once saturated, the inductance of the circuit drops dramatically. Taking account other phenomena like hysteresis, the resultant waveform on the secondary circuit coupled by a saturated core is highly distorted and full of harmonics. Depending on the level of saturation and the capability of the measuring device to process the distorted waveform (harmonics) the measured current on the secondary is much smaller than the corresponding RMS value present in the primary.

\subsection{Offset}

Basic principle of electromechanical conversion states that the output on the secondary is related to the variation of the coupled magnetic flux. It explains why a regular CT and transformers can't operate on DC. Although the DC current is not converted into secondary current, it does generates magnetic flux and thus contribute to core saturation. Theoretically a core fully saturated by a DC current has no output (DC current causes no variation of coupled magnetic flux through time). Asymmetrical faults (like phase-phase) may generate DC components contributing to CT saturation (That's where the 20x criteria on CT sizing came from Equation (1)). Although, resulting in non-linear output, the saturation of a CT magnetic core has a different characteristic from other saturation phenomena observed on systems like audio amplifiers. The bottom line is that the current waveform on the secondary of a saturated CT may be diverse depending on what components are present on the primary current and also on the burden wired to the secondary circuit of the CT. CT saturation leads to protection devices maloperation as the measured current does not correspond to the actual current on the system. 


\subsection{Burden on CT Secondary}

All other factors being equal, a CT with a higher total secondary burden of the same power factor will have a shorter time-to-saturation. This is because the higher burden demands a higher voltage at a given current and the flux is proportional to the voltage. For two impedances of the same magnitude, the one with the more inductive component (lower $\mathrm{pF}$ ) will give a longer time-tosaturation because the inductance has low impedance to the DC offset current, reducing the burden voltage drop and associated flux. A second characteristic of inductive burdens is their tendency to saturate at high secondary currents. The effect is to reduce the burden volt drop, which reduces the flux and increases the time-to-saturation. When an inductive burden is added to a CT circuit, the magnitude of the overall ohmic burden is not substantially increased (even if saturation of the burden is ignored) because the impedances are added as vectors rather than as scalars.

\section{SVM Based Proposed Method}

This article presents a saturation of current transformer and its classification with various aspects. Various level of CT saturation i.e. low, medium and heavy by considering different factors are analyzed for classification of unsaturated and saturated cases. Authors have suggested SVM based method to discriminate saturation of current transformer and compared with ANN technique.

Figure 1 shows the schematic block diagram of the proposed scheme. Initially, the SVM is configured using training data set; samples of current signals of all the lines are acquired by data acquisition system. After configuring the SVM, samples of post fault CT secondary current signals are utilized to formulate the feature vectors with the help of testing data set. Soon after, these feature vectors are used as an input to SVM classifier. The output of the SVM is used to identify the saturated and unsaturated case.

SVM is a relatively new computational learning method based on the statistical learning theory. It is used as a very powerful tool to solve the classification problems. It tries to find out an 'optimal hyper-plane' to separate the data points according to their classes such that the separation between the classes is maximum. A fault classification task usually involves training and testing of some data instances. Each instance in the training set contains one "target value" (class labels) and several "attributes" (features). The goal of the SVM is to produce a model that predicts the target value of data instances 


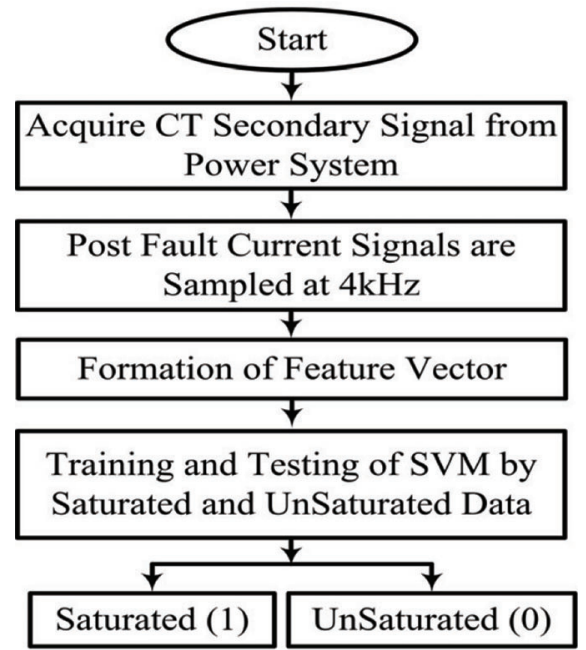

Figure 1 Proposed SVM based algorithm to detect CT saturation.

in the testing set, which are given only the attributes [22]. The output of SVM, the value ' 1 ' and ' 0 ', denotes the saturated and unsaturated current signal respectively. The SVM technique has been implemented in MATLAB environment using Lib-SVM toolbox [23].

For accurate and efficient SVM classification, it is essential to implicitly map the input vector into a high dimensional feature space using a non-linear transformation. In practice, this non-linear transformation is accomplished indirectly by using the so called kernel functions, which is defined by,

$$
K\left(x_{i}, x_{j}\right)=\Phi\left(x_{i}\right)^{T} \Phi\left(x_{j}\right)
$$

The most commonly used kernel functions are as under:

1. Linear: $K\left(x_{i}, x_{j}\right)=\left(x_{i}\right)^{T}\left(x_{j}\right)$

2. Polynomial: $K\left(x_{i}, x_{j}\right)=\left\{y\left(x_{i}\right)^{T}\left(x_{j}\right)+r\right\}^{d}, y>0$

3. Radial Basis Function (RBF): $K\left(x_{i}, x_{j}\right)=\exp \left(-g\left|x_{i}-x_{j}\right|^{2}\right), \quad g>0$

4. Sigmoid: $K\left(x_{i}, x_{j}\right)=\tanh \left\{y\left(x_{i}\right)^{T}\left(x_{j}\right)+r\right\}$

Where, $\mathrm{y}, \mathrm{r}$ and $\mathrm{d}$ are kernel parameters.

Referring to many literatures, it has been observed by the authors that Radial Basis Function (RBF) kernel is the first choice for training as it nonlinearly maps samples into higher dimensional space most effectively among all types of kernels used [24]. 


\section{System Study and Data Generation}

A three phase $11 \mathrm{kV}, 50 \mathrm{~Hz}, 315$ MVA Thevenin's equivalent generator is connected to $11 / 220 \mathrm{kV}, 350 \mathrm{MVA}$, YY connected transformer as shown in Figure 2. A $220 \mathrm{kV}$ transmission line $(80 \mathrm{~km})$ is originated from $220 \mathrm{kV}$ bus and connected to infinite bus, on which 1200/1 Ampere CT is connected to acquire the current signal during various abnormal situations. Various saturated and unsaturated cases are simulated using PSCAD/EMTDC software and validated using SVM classifier implemented in MATLAB. To validate the proposed algorithm, the CT located on transmission line (Figure 2) is analyzed which uses Jiles - Atherton model available in PSCAD/EMTDC software package [25].

Table 1 shows different parameter values which have been chosen in this work to generate CT saturation conditions. Table 1 also shows breakup of total 3600 saturated cases, among them 2000 data are consider as training data and remain 1600 data are taken for testing.

Similarly, as shown in Table 2, unsaturated CT secondary data are generated by system and fault parameter variations. Table 2 also shows bifurcation of total 720 unsaturated data, among them 480 data are considering as training and remaining 240 data are consider as testing of the proposed algorithm. Authors have generated these data by simulating Figure 2 in PSCAD/EMTDC software. The data being generated using multirun block available in PSCAD for parameter variation mentioned in Tables 1 and 2.

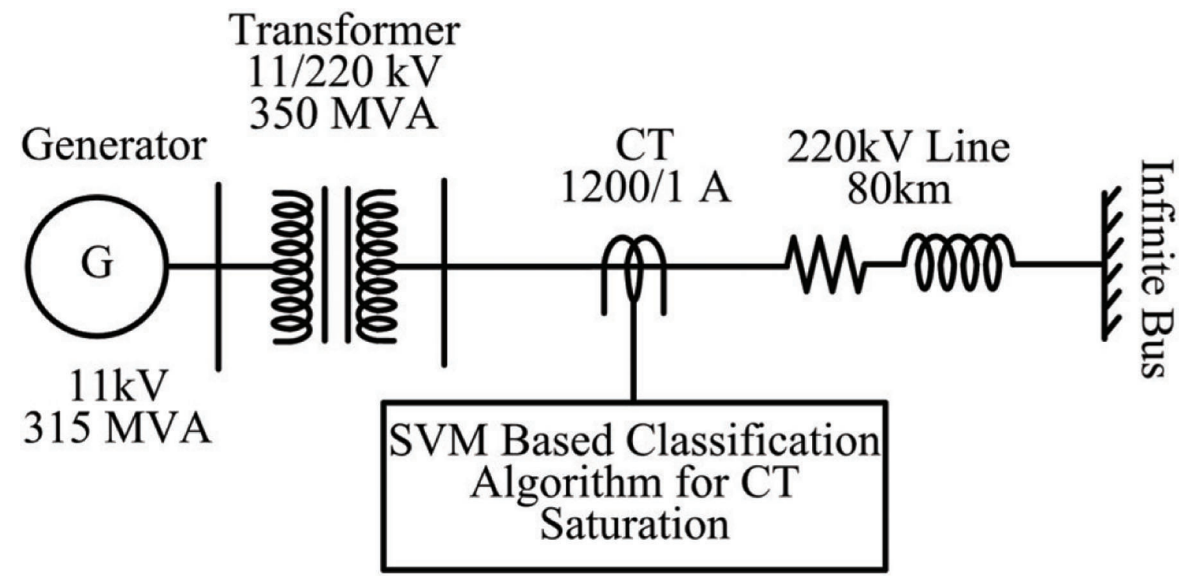

Figure 2 Single line diagram of power system. 
Table 1 CT Saturated Data Collection for Training and Testing of SVM

\begin{tabular}{|c|c|c|c|}
\hline \multirow[b]{2}{*}{ Sr. } & \multirow[b]{2}{*}{ Parameter } & \multicolumn{2}{|c|}{ Saturated Data } \\
\hline & & Total No's of Data & Training Data \\
\hline \multirow[t]{2}{*}{1} & Fault Inception & $00,300,450,900,1350,2700$ & $00,300,900,1350,2700$ \\
\hline & Angle (FIA) & $(6)$ & $(5)$ \\
\hline \multirow[t]{2}{*}{2} & Secondary & $5 \Omega, 8 \Omega, 10 \Omega, 15 \Omega, 25 \Omega$ & $5 \Omega, 8 \Omega, 10 \Omega, 15 \Omega, 25 \Omega$ \\
\hline & Burden & $(5)$ & $(5)$ \\
\hline 3 & Remnant Flux & $20 \%, 30 \%, 50 \%, 70 \%, 85 \%, 95 \%$ & $\begin{array}{c}20 \%, 30 \%, 70 \%, 85 \%, 95 \% \\
(5)\end{array}$ \\
\hline 4 & $\begin{array}{l}\text { Signal to Noise } \\
\text { (SNR) }\end{array}$ & $10 \mathrm{db}, 20 \mathrm{db}, 30 \mathrm{db}, 40 \mathrm{db}$ & $10 \mathrm{db}, 20 \mathrm{db}, 30 \mathrm{db}, 40 \mathrm{db}$ \\
\hline 5 & $\begin{array}{l}\text { X/R Ratio of } \\
\text { Line }\end{array}$ & $7,8,10,12,15$ & $\begin{array}{l}7,10,12,15 \\
\quad(4)\end{array}$ \\
\hline \multicolumn{2}{|c|}{ Total } & $6 * 5 * 6 * 4 * 5=3600$ Total Data & $5 * 5 * 5 * 3 * 4=2000$ Training data \\
\hline
\end{tabular}

Table 2 CT Unsaturated Data Collection for Training and Testing of SVM

\begin{tabular}{|c|c|c|c|}
\hline \multirow[b]{3}{*}{ Sr. } & \multirow{3}{*}{$\begin{array}{l}\text { Parameter } \\
\text { Variation }\end{array}$} & \multicolumn{2}{|c|}{ Unsaturated Data } \\
\hline & & & \\
\hline & & Total No's of Data & Training Data \\
\hline \multirow[t]{2}{*}{1} & Fault Inception & $00,300,450,900,1350,2700$ & $00,300,450,1350,2700$ \\
\hline & Angle (FIA) & $(6)$ & $(5)$ \\
\hline 2 & $\begin{array}{l}\text { Secondary Burden } \\
\text { (very low) }\end{array}$ & $0 \Omega, 0.05 \Omega, 0.1 \Omega$ & $\begin{array}{c}0 \Omega, 0.05 \Omega, 0.1 \Omega \\
\text { (3) }\end{array}$ \\
\hline 3 & Remnant Flux & $\begin{array}{c}0 \%, 10 \% \\
(2)\end{array}$ & $\begin{array}{l}0 \%, 10 \% \\
\text { (2) }\end{array}$ \\
\hline 4 & $\begin{array}{l}\text { Signal to Noise } \\
\text { (SNR) }\end{array}$ & $10 \mathrm{db}, 20 \mathrm{db}, 30 \mathrm{db}, 40 \mathrm{db}$ & $10 \mathrm{db}, 20 \mathrm{db}, 30 \mathrm{db}, 40 \mathrm{db}$ \\
\hline 5 & X/R Ratio of Line & $\begin{array}{c}7,8,10,12,15 \\
(5)\end{array}$ & $\begin{array}{c}7,8,10,15 \\
(4)\end{array}$ \\
\hline \multicolumn{2}{|c|}{ Total } & $6 * 3 * 2 * 4 * 5=720$ Total Data & $5 * 3 * 2 * 4 * 4=480$ Training data \\
\hline
\end{tabular}

\section{Result Analyses and Discussion}

In order to achieve accurate CT saturated condition identification, proper training of SVM is extremely important. For this purpose, out of the total of $3600+720=4320$ cases, $2000+480=2480$ fault cases have been chosen for training with different system parameters as given in Tables 1 and 2. Remaining cases $(1600+240=1840)$ have been used for testing $\&$ validation 
of the proposed technique. The fault classification accuracy $(\eta)$ is given by,

$$
\eta=\frac{\text { Number of correct fault classification casess }}{\text { Total Number of test cases }(1840)} \times 100 \%
$$

Once the training cases have been identified among all the simulated cases, the next step is to train SVM with the most optimal parametric settings. To achieve this, type of kernel function to be used for SVM with its associated parameters must be decided. It has been observed that with the optimized kernel parameters i.e. $\mathrm{g}=0.0032$ and $\mathrm{C}=1000$, the Gaussian RBF kernel gives the highest accuracy of $99.67 \%$. These regularization parameters are selected by trial and error method for which it gives highest classification accuracy.

In order to test effectiveness of the proposed scheme under varying system conditions, a large numbers of simulation cases have been generated. Different parameter values which have been chosen to produce the transient response of $\mathrm{CT}$ are remanence flux density, burden resistance and presence of DC offset \& noise. Considering these all parameter values, around 4320 simulations cases were generated and the effectiveness of the proposed scheme was verified for all these test cases (1840). Figure 3 shows waveform of saturated and unsaturated CT secondary current in presence of decaying DC component along with higher value of burden resistance $(10 \Omega)$. Conversely, Figure 4 shows same waveforms without DC component and having moderate CT saturation.

The amount of remanent flux in the core depends on factors such as magnitude of primary current, the burden on secondary circuit and the

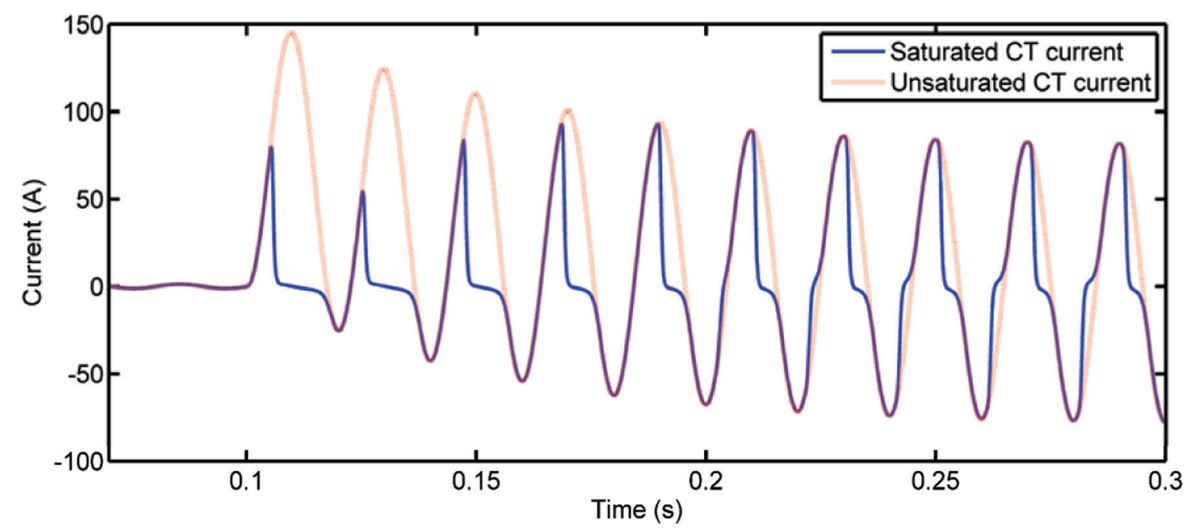

Figure 3 Waveform of CT secondary current with saturation and without saturation including $\mathrm{DC}$ component for burden resistance $=10 \Omega$. 


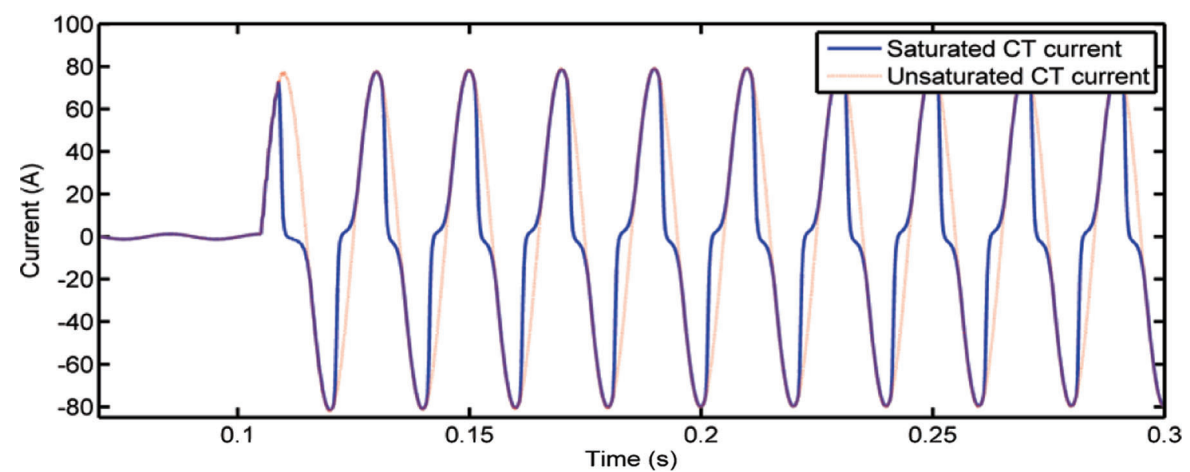

Figure 4 Waveform of CT secondary current with light saturation and without DC component for burden resistance $=5 \Omega$.

amplitude \& time constant of decaying DC component. Depending upon the direction of flux setup in the core during the energization of $\mathrm{CT}$ in presence of remanent flux, a large part of secondary current of CT may saturate. In this situation, the performance of protective class CT is influenced by this remanence or residual magnetism and may reach up to $90 \%$ of the saturation flux. Figure 5 show the CT secondary currents during fault applied at FIA = 135 degree with $0.5 \Omega$ burden resistance with $50 \%$ remanent flux density. This remanent flux density was set in the core of CT prior to inception of fault.

To evaluate the proposed algorithm, acquired current signals from PSCAD/EMTDC software are polluted with white Gaussian noise by considering different Signal-to-Noise Ratios (SNR) in MATLAB environment.

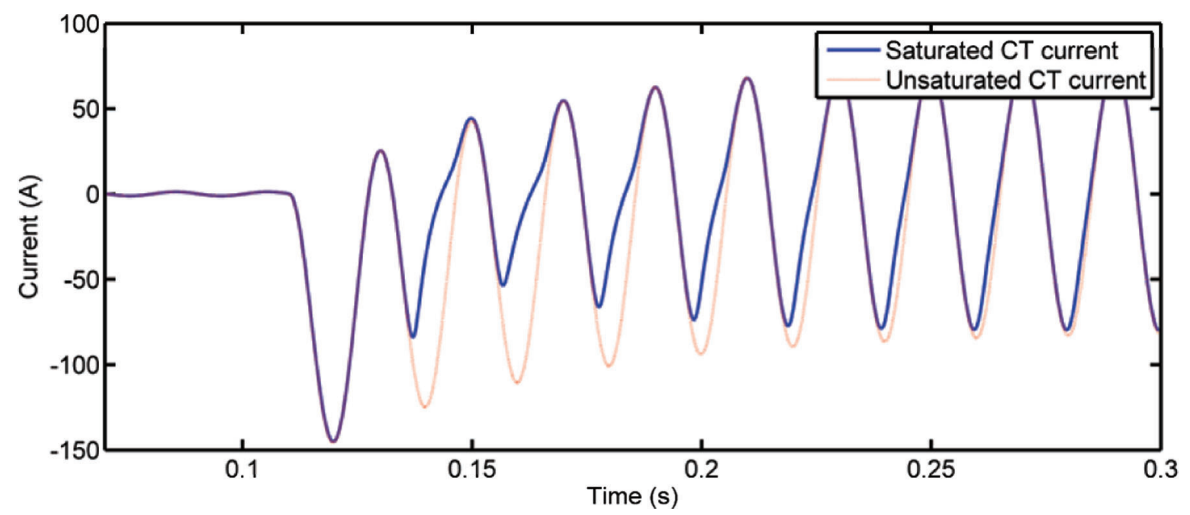

Figure 5 Waveform of CT secondary currents with 50\% remanence flux. 
The SNRs are set to $10 \mathrm{db}$ to $40 \mathrm{~dB}$ to pollute the original current signals. Figure 6 show the saturated and unsaturated CT secondary signals with $0.5 \Omega$ burden resistance and SNR $=40 \mathrm{db}$.

In this study, the cases classified correctly and incorrectly are denoted as True Positive (TP) and True Negative (TN) respectively. The performance evaluation of the proposed scheme has been carried out for different types of CT secondary signals with varying system parameters over a total of 1840 test cases and is presented in Tables 3 and 4 . The results have been analyzed for both scenarios: (i) with saturation considered and (ii) without CT saturation considered. It has been observed from Table 3 that the proposed scheme is capable to discriminate between saturated and unsaturated condition of CT giving a classification accuracy of $99.68 \%$ and $99.5 \%$, respectively. Also, an overall fault classification accuracy of $99.67 \%$ is obtained which shows the effectiveness of the proposed scheme in practical scenario.

Table 4 demonstrate the performance of the proposed scheme for CT saturation during different type of parameter variations. It has been observed from Table 4 that the proposed scheme correctly identifies CT saturation conditions

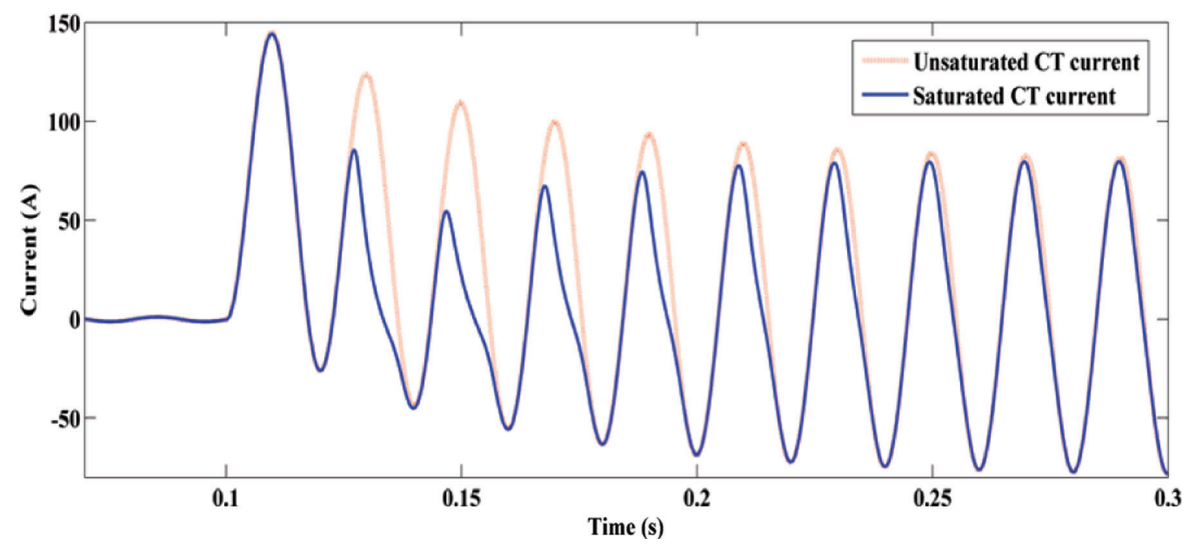

Figure 6 Waveform of CT secondary currents during SNR $=40 \mathrm{db}$.

Table 3 Overall classification accuracy with and without CT saturation

\begin{tabular}{|c|c|c|c|c|c|}
\hline \multirow[b]{2}{*}{ Sr. } & \multirow[b]{2}{*}{ CT Condition } & \multirow[b]{2}{*}{ Total } & \multicolumn{3}{|c|}{ SVM } \\
\hline & & & TP & TN & Efficiency (\%) \\
\hline 1 & Saturated (1) & 1600 & 1595 & 5 & 99.68 \\
\hline 2 & Unsaturated (0) & 240 & 239 & 1 & 99.5 \\
\hline \multicolumn{2}{|c|}{ Total } & 1840 & 1834 & 6 & 99.67 \\
\hline
\end{tabular}


Table 4 Case wise classification accuracy for CT saturated data

\begin{tabular}{llcccc}
\hline & & \multicolumn{3}{c}{ SVM } \\
\cline { 3 - 5 } Sr. & Different Cases & Test Data & TP $(1)$ & TN (0) & Efficiency (\%) \\
\hline 1 & Fault Inception Angle (FIA) & 348 & 348 & 0 & 100 \\
2 & Secondary Burden & 348 & 347 & 1 & 99.71 \\
3 & Remnant Flux & 348 & 347 & 1 & 99.71 \\
4 & Signal to Noise (SNR) & 278 & 276 & 2 & 99.28 \\
5 & X/R Ratio & 278 & 277 & 1 & 99.64 \\
Total & 1600 & 1595 & 5 & 99.68 \\
\hline
\end{tabular}

with very high overall fault classification accuracy. The results confirm the effectiveness of the proposed SVM based technique for discrimination of CT secondary current with \& without saturation. Result demonstrated in Tables 3 and 4 confirm that a Statistical Learning Theory (SLT) based SVM provides non-linear classification which can be proved to be a more powerful method compare to neural network and fuzzy based methods. The same has been observed as an outcome of the result analysis presented in this section. Also, it does not require any post processing of the signal (phasor computation, frequency analysis etc.) hence, can be considered to be more accurate and faster.

\section{Conclusions}

Maloperation in relaying schemes are generated due to CT saturation. So, power system protective schemes must be equipped with appropriate mean to correctly identify saturation condition of CT. In this article, a Support Vector Machine (SVM) based algorithm is proposed to distinguish CT saturation state under its various operating conditions. The proposed scheme effectively discriminates between unsaturated and saturated current signal. Post fault current signals of transmission line CT secondary are given as an input to SVM. It has been observed that the proposed scheme gives an overall fault classification accuracy of more than $99 \%$. Around 1840 simulation cases have been considered for validation of the suggested scheme among the total simulated dataset consisting of 4320 cases with different fault and system parameters variations. These cases are generated by considering parameters such as remanence flux, Fault Inception Angle (FIA), burden resistance and presence of DC offset \& Signal to Noise Ratio (SNR). The proposed technique perfectly categorizes different saturation cases with highest efficiency and hence, found to be superior to the existing schemes. 


\section{Acknowledgment}

This work is supported by Department of Science and Technology under SERB (India) project no. EMR/2016/006041.

\section{References}

[1] Hooshyar, A., Sanaye Pasand, M., and El Saadany E. F. (2013). CT Saturation detection based on wave shape properties of current difference functions. IEEE Trans. Power Deliv. 28, 2254-2263.

[2] Hooshyar, A., Sanaye Pasand, M., Davarpanah, M. (2012). Development of a new derivative-based algorithm to detect current transformer saturation. IET Gener. Transm. Distrib. 6, 207-217.

[3] Chothani, N. G., and Bhalja, B. R. (2014). New algorithm for current transformer saturation detection and compensation based on derivatives of secondary currents and newton's backward difference formulae. IET Gener. Transm. Distrib. 8, 841-850.

[4] Kang, Y. C., Ok, S. H., Kang, S. H., and Crossley, P. A. (2004). Design and evaluation of an algorithm for detecting current transformer saturation. IEE Proc. Gen. Trans. Distrib. 151, 27-35.

[5] Kang, S.-H., Kim, M.-S., Nam, S.-R., Shin, J. H., and Jung, J.-J. (2014). "A CT saturation detection algorithm based on wavelet transformation developments in power system protection," in Proceedings of the 12th IET International Conference on DPSP, Copenhagen, 1-4.

[6] Bak, D. J., Dong, X. Z., Wang, B., Shin, S. X., and Rebizant, W. (2012)"New method of detection of current transformer saturation," in Proceedings of the 11th International Conference on Developments in Power Systems Protection (DPSP) (Red Hook, NY: Curran Associates, Inc), 1-5.

[7] Hooshyar, A., Sanaye-Pasand, M., and El Saadany, E. F. (2013) CT Saturation detection based on wave shape properties of current difference functions. IEEE Trans. Power Deliv. 28, 2254-2263.

[8] Hajipour, E., Vakilian, M., and Sanaye Pasand, M. (2015). Current transformer saturation compensation for transformer differential relays. IEEE Trans. Power Deliv. 30, 2293-2302.

[9] Ji, T. Y., Wu, Q. H., Tang, W. H., and Jiang, L. (2011). "A morphological scheme for the correction of CT saturation waveforms," in Proceedings of the IEEE Power and Energy Society General Meeting, Chicago, IL, $1-7$. 
[10] Badrkhani Ajaei, F., Sanaye-Pasand, M., Davarpanah, M., Rezaei-Zare, A., and Iravani, R. (2011). Compensation of the current-transformer saturation effects for digital relays. IEEE Trans. Power Deliv. 26, 2531-2540.

[11] Shi, D. Y., Buse, J., Wu Q. H., and Jiang L. (2010) "Fast compensation of current transformer saturation," in Proceedings of the IEEE PES Innovative Smart Grid Technologies Conference Europe (ISGT Europe), Kollam, 1-7.

[12] Esmail, E. M., Elkalashy, N. I., Kawady, T. A., Taalab, A. M. I., and Lehtonen, M. (2015). Detection of partial saturation and waveform compensation of current transformers. IEEE Trans. Power Deliv. 30, $1620-1622$.

[13] Villamagna, N., and Crossley, P. A. (2006). A CT saturation detection algorithm using symmetrical components for current differential protection. IEEE Trans. Power Deliv. 21, 38-45.

[14] Dos Santos, E. M., Cardoso, G., Farias, P. E., and de Morais, A. P. (2013). CT saturation detection based on the distance between consecutive points in the plans formed by the secondary current samples and their differencefunctions. IEEE Trans. Power Deliv. 28, 29-37.

[15] Chothani, N. G., and Sharma, V. (2015). "A new method for CT saturation detection using secondary current envelope detector," in Proceedings of the International Conference on Recent Developments in Control, Automation and Power Engineering (RDCAPE), Noida, 1-6.

[16] Solak, K., Rebizant, W., and Klimek, A. (2012). Fuzzy adaptive transmission-line differential relay immune to ct saturation. IEEE Trans. Power Deliv. 27, 766-772.

[17] Hooshyar, A., and Sanaye-Pasand, M. (2011). CT saturation detection based on waveform analysis using a variable-length window. IEEE Trans. Power Deliv. 26, 2040-2050.

[18] Hooshyar, A., and Sanaye-Pasand, M. (2015). Wave shape recognition technique to detect current transformer saturation. IET Gen. Trans. Distrib. 9, 1430-1438.

[19] Kappenman, J. G., Albertson, V. D., and Mohan, N. (1981). Current transformer and relay performance in the presence of geomagneticallyinduced currents. IEEE Trans. Power Apparatus Syst. 100, 1078-1088.

[20] Schettino, B. M., Duque, C. A., Silveira, P. M., Ribeiro, P. F., and Cerqueira, A. S. (2014). A new method of current-transformer saturation detection in the presence of noise. IEEE Trans. Power Deliv. 29, 1760-1767. 
[21] (2005). Introduction to PSCAD/EMTDC. Winnipeg, MB: Manitoba HVDC Research Centre, Inc.

[22] Cristianini, N., and Shawe-Taylor, J. (2000). An Introduction to Support Vector Machines and Other Kernel-Based Learning Methods, 1st Edn. Cambridge: Cambridge University Press.

[23] Chang, C.-C., and Lin, C.-J. (2016). LIBSVM - A Library for Support Vector Machines. Available at: http://www.csie.ntu.edu.tw/ $/$ cjlin/libsvm/

[24] Chothani, N. G., Bhalja, B. R., and Parikh, U. (2011). New fault zone identification scheme for busbar using support vector machine. Proc. IET Gener. Trasmn. Distrib. 5, 1073-1079.

[25] Annakkage U. D., McLaren P. G., Dirks E., Jayasinghe R. P., and Parker A. D. (2000). A current transformer model based on the Jiles - Atherton theory of ferromagnetic hysteresis. IEEE Trans. Power Deliv. 15, 57-61.

\section{Biographies}

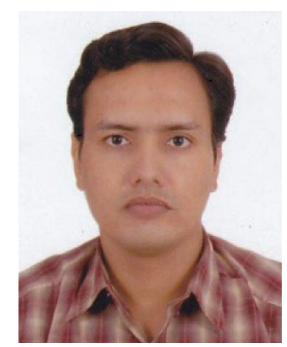

N. G. Chothani is an Associate Professor in the Department of Electrical Engineering, ADIT, New Vallabh Vidhyanagar, India. He received B.E. degree from Saurashtra University, Rajkot, Gujarat in 2001. He received Master degree in power system and the Ph.D. degree in electric engineering from the Sardar Patel University, Vallabh Vidhyanagar, Anand, India in 2004 and 2013, respectively. He has published several research paper and national and international referred journals. His research interests include power system protection, simulation, power system modeling and artificial intelligence. 


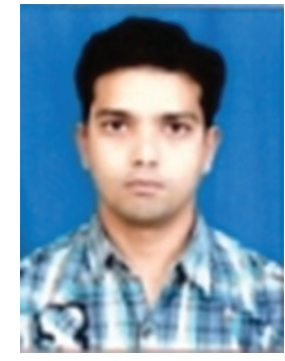

D. D. Patel is an Assistant Professor in the Department of Electrical Engineering, Government Engineering College, Bharuch, Gujarat, India. He received B.E. degree from North Gujarat University, Patan, Gujarat in 1999 and Master degree in power system from the Sardar Patel University, Vallabh Vidhyanagar, Anand, India in 2002. Currently he is pursuing Ph.D. at Sardar Vallabhbhai National Institute of Technology, Surat, India. His field of research is power transformer protection.

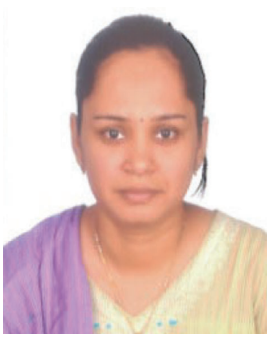

K. D. Mistry is an Assistant Professor in the Department of Electrical Engineering, Sardar Vallabhbhai National Institute of Technology, Surat, Gujarat, India. She received B.E. degree in Electrical Engineering and Master degree in power system from the Sardar Patel University, Vallabh Vidhyanagar, Anand, India in 2004 and 2006 respectively. She received Ph.D. degree in electrical engineering from Sardar Vallabhbhai National Institute of Technology, Surat, India in 2015. Her research interest is in optimization techniques and analysis of power system, Power system protection. 
